\title{
COVID-19'un Halk Sağlığı Açısından Yarattığı Rahatsızlıkların Çok Boyutlu Faktör Kümelerinin Tahmini
}

\author{
Adnan Mazmanoğlu $\mathbf{u}^{*}$ \\ 1* Toros Üniversitesi, Mühendislik Fakültesi, Endüstri Mühendisliği Bölümü, Mersin, Türkiye (ORCID: 0000-0002-8756-8429), adnan.mazmanoglu@toros.edu.tr
}

(İlk Geliş Tarihi 2 Mart 2021 ve Kabul Tarihi 20 Eylül 2021)

(DOI: 10.31590 /ejosat.889609)

ATIF/REFERENCE: Mazmanoğlu, A. (2021). COVID-19'un Halk Sağlığı Açısından Yarattığı Rahatsızlıkların Çok Boyutlu Faktör Kümelerinin Tahmini. Avrupa Bilim ve Teknoloji Dergisi, (27), 508-517.

Öz

Bu çalı̧̧ma için, Türkiye'de Mart 2020 tarihinden başlayan pandemi sürecinde Web tabanlı rastgele (random) örneklem yöntemi kullanılarak yapılmış kesitsel bir anket çalışması yapılmıştır. İlk bakışta Anket çalışması dört ana unsur (kategori) biçiminde olduğu düşünülebilir: (1) Katılımcıların demografik özellikleri, (2) Salgının risk ve tehlike algısı, (3) Salgından bireysel, toplumsal korunma ve aşıya yaklaşım, (4) Salgının fizyolojik ve psikolojik etkisinin incelenmesi şeklinde olduğu söylenebilir. Basitçe verilerden istatistiksel modelleme analizi yapmadan önce COVID-19 Pandemisinin toplumda çok yönlü ve karmaşık bir bask altında kalma psikolojisinin hâkim olduğunu kolayca gözleyebildik. Böyle bir kaniya ve/veya düşmemizi güçlendirecek bilgiye ulaşmak için "çok değişkenli bir istatistiksel model olan faktör analizi yöntemleriyle" çalışmayı uygun bulduk. Pandemi nedeniyle yaşanabilecek ekonomik sıkıntılar ve iş bulma kaygısı gerek COVID-19'a yakalanma gerekse interaktif eğitim sistemine uyum kaygılarının önüne geçmiş olup toplumda psikolojik ve fizyolojik rahatsızlıkların ya oluşmasına ya da mevcut rahatsızlıkların ortaya çıkmasına temel oluşturmuş olduğu araştırılmış olsa da bir sosyolojik rahatsızlıklar kümesel yakın korelasyonlu faktörlerin ortaya çıkmasıyla psikologlara önemli bir etki yaratacağı kesindir (yüksek bir olasılık eşiğiyle). İzolasyon sürecinde halk sağlığı üstüne etkisinin belirlenmesine yönelik kesitsel tipte bir anket çalışması yapıldı. Anket, katılımcıların WhatsApp ve Twitter hesaplarına web sitesinden yayınlandı. Anket, katılımın gönüllü niteliği, anonimlik, gizlilik beyanları ve anket soruları ile ilgili kısa bir giriş içermekteydi. Katılımcılar, gönüllü olarak katılma isteklerini teyit etmek amaciyla "Evet veya Hayır" seçeneğine göre anketi doldurmaya yönlendirildi. Çalışma, 49 ilde (toplam 81 il olduğu düşünüldüğünde yaklaşık \%60'llk bir katılım) elektronik ortamda rastgele seçilmiş 931 katılımcının oluşturduğu örneklemle gerçekleştirildi. Örneklemin yaş ortalaması 41 ve ranjı 11-86 aralığındadır. Verilen 4 sınıftan (kategoriden) oluşan anketin sorularından F. A. 'i için 68 değişken ataması yapılmıştır.

\section{Estimation of Factor Clusters from Multidimensional Modeling of COVID-19 Diseases in Terms of Public Health}

\begin{abstract}
For this study, the pandemic period starting from March 2020, Turkey Web-based random (random) sampling method is carried out using a cross-sectional survey was conducted. At first glance, the survey can be thought to be in the form of four main elements (categories): (1) The demographic characteristics of the participants, (2) The risk and danger perception of the epidemic, (3) Individual, social protection from the epidemic and the approach to vaccination, (4) The physiological and psychological impact of the epidemic. It can be said to be examined. We could easily observe that the COVID-19 Pandemic is dominated by a multifaceted and complex psychology of oppression in the society, simply before performing statistical modeling analysis from the data. We found it appropriate to work with "factor analysis methods, which is a multivariate statistical model" in order to reach such an opinion and / or information that will strengthen our falling. Although it has been investigated that the economic difficulties and the anxiety of finding a job due to the pandemic have prevented both the concerns of catching COVID-19 and the adaptation to the interactive education system and it has been investigated that they have formed the basis for the occurrence of psychological and physiological disorders in the society or the emergence of existing diseases, sociological disorders are closely correlated.The emergence of factors is certain to have a significant impact on psychologists (with a high probability threshold). A cross-sectional survey study was conducted to determine the effect on public health during the isolationprocess. The survey was published on the website of the participants' WhatsApp and Twitter accounts. The survey contained a brief introduction to the voluntary nature of the participation, anonymity, privacy statements, and survey
\end{abstract}


questions. Participants were prompted to fill out the questionnaire according to the "Yes or No" option to confirm their willingness to participate voluntarily. The study was carried out with a sample of 931 randomly selected participants in 49 provinces (approximately $60 \%$ participation considering that there are 81 provinces in total). The average age of the sample is 41 and the range is between 11 and 86. From the questions of the questionnaire consisting of 4 classes (categories) given, 68 variables were assigned for F. A

Keywords: Publichealth, Coronavirus, Pandemic, Principal Compenent Analysis, Factor Analysis.

\section{Giriş}

COVID-19 salgın süreci "çok değişkenli çok boyutlu" etkilerin yaşandığı bir süreç olmakta. Bu "çok değişkenli çok boyutlu" bir rastlantısal sürecin anlamlı olabilmesi için çok boyutlu çözüm teknikleriyle bir istatistiksel model kurmak zorundayız. Zira "belirsizliğin" olduğu araştırmalarda oluşturulan verilerin çözümlemesi ya da "çok boyutlu" çözümleme yöntemlerinin bir bölümü yenilenir, yenileri de sürekli ortaya çıkarılırken, bunları sınıflamak oldukça güçtür. Bunun da değişkenlerle aralarındaki ilişkinin yarattığı sorunların niteliğine bağlı olduğunu belirtebiliriz. Bu arada türlü yöntemlere örnek olarak "faktör", "regresyon", "korelasyon", "varyans", "path" analizlerini," kümeler arası çözümleme" ve "discriminant (ayrımcı)" çözümlemeyi, aralarındaki ilişkilerle gösteriyorlar. Biz de bilgisayarların very işleme hızının gelişmesi, değişik türdeki (nitel-nicel) alanlarda anketlerin düzenlenmesi olgularına dayanarak istatistiğin önemli bir bölümünü oluşturan yukarıda saydığımız çözümleme yöntemleri; "başlıca bileşenler çözümlemesi" ve "kümeler arası çözümleme" yöntemlerinin temelini oluşturur (Spearman-Pearson). Bilhassa bizim yaptığımız anketin konusunu da içeren "Salgın (pandemi)" dan doğan olumsuz sağlık bozucu, sosyolojik, psikolojik, tıp, biyoloji, çevre kirliliği vb. hemen hemen tüm bilim dallarını ilişkilendiren, çok değişkenli istatistik tekniklerinden en popüler olanlardan "Faktör analizi (factor analysis)" çok boyutlu ölçekleme için uygun bir yöntem olarak sayılabilir.

\section{Materyal ve Metot}

\subsection{Faktör Analizi}

Faktör analizin değişkenleri bir gözlenen olaydır. Buradaki değişken, belirli bir sonucu doğuran veya etkileyen her olay bir değişkendir. Etkileyici elemanlar bölünüp parçalanarak bazıları birleştirilerek olaylar grubu ortaya çıkarılır. Sözü edilen gruplar Fktör analizinde değişkenler kümesini oluşturur. Tahminleme örnek vasıtasıyla yapılır. Yapı şudur: F.A.' inde değişkenler incelenecek ana kütleye bağlı olmalı. Yani bir değişken diğerinden etkilenmemeli, bir değişkenin sonucu başka bir değişkeni oluşturmamalı. İlk bakışta her değişkenin eşit ağırlığı olmalıdır. Bir olay veya sonuca etki eden elemanların içinde önemli olanların (en etkili olanın) bulunması, önem derecesinin saptanması için bu analiz tekniğinden yararlanarak değişkenleri bazı döndürme teknikleriyle küçülterek ve sayı ile ifade etmek gerekir. Özetle amaç: F.A. kullanılarak değişkenler arasındaki karşılıklı bağlılığın kökünü ortaya koymaktır. Başka bir deyişle, en az bilgi kaybıyla, büyük hacimdeki verilerden özetlenmiş ve yeni bir yapı içerisinde (Hotelling, 1960) verileri elde etmektir. $\mathrm{Bu}$ yeni biçim (form), çok sayıdaki değişkenin verilerine göre şekillenmesinden oluşur. Sonuç olarak F.A. 'inin amacı verilerin yapısını tanımlamak, verileri özetlemek, sayılarını yönetebilir ve üstünde çalışabilir, uygun bir sayıya düşürmektir. Bu özellik bilhassa çok değişkenli veri grupları için çok önemli ve değişkenlerin "nitel özellikli” olmasını da çözmüş olmaktadır.
Çalışmada IBM SPSS Statistics Version.24 yazılımı ile variables (değişkenler) orijinal olarak aşağıda Var kısaltması ile tanımlanmıştır

\section{GET}

FILE='C: \Users\user\Documents \Untitled1-ADNAN-

MÜZEYYEN.sav-VERILER.sav'.

DATASET NAME DataSet1 WINDOW=FRONT.FACTOR

/VARIABLES VAR1 VAR2 VAR3 VAR4 VAR5 VAR6 VAR7 VAR8 VAR9 VAR10 VAR11 VAR12 VAR13 VAR14 VAR15 VAR16 VAR17 VAR18 VAR19 VAR20 VAR21 VAR22 VAR23 VAR24 VAR25 VAR26 VAR27 VAR28 VAR29 VAR30 VAR31 VAR32 VAR33 VAR34 VAR35 VAR37 VAR36 VAR38 VAR39 VAR40 VAR41 VAR42 VAR43 VAR44 VAR45 VAR46 VAR47 VAR48 VAR49 VAR50 VAR51 VAR52 VAR53 VAR54 VAR55 VAR56 VAR57 VAR58 VAR59 VAR60 VAR61 VAR62 VAR63 VAR64 VAR65 VAR66 VAR67 VAR68

/MISSING LISTWISE

/ANALYSIS VAR1 VAR2 VAR3 VAR4 VAR5 VAR6 VAR7 VAR8 VAR9 VAR10 VAR11 VAR12 VAR13 VAR14 VAR15 VAR16 VAR17 VAR18 VAR19 VAR20 VAR21 VAR22 VAR23 VAR24 VAR25 VAR26 VAR27 VAR28 VAR29 VAR30 VAR31 VAR32 VAR33 VAR34 VAR35 VAR37 VAR36 VAR38 VAR39 VAR40 VAR41 VAR42 VAR43 VAR44 VAR45 VAR46 VAR47 VAR48 VAR49 VAR50 VAR51 VAR52 VAR53 VAR54 VAR55 VAR56 VAR57 VAR58 VAR59 VAR60 VAR61 VAR62 VAR63 VAR64 VAR65 VAR66 VAR67 VAR68

/PRINT INITIAL CORRELATION SIG KMO EXTRACTION ROTATION

/FORMAT SORT

/PLOT EIGEN

/CRITERIA MINEIGEN(1) ITERATE(25)

/EXTRACTION PC

/CRITERIA ITERATE(25)

/ROTATION VARIMAX

$/$ METHOD $=$ CORRELATION.

F ACTOR ANALYSIS _........

\section{Araştırma Sonuçları ve Tartışma}

\subsection{Veriyi Sınama}

Değişkenlere verilen yanıtlara uygun olarak bellekte (byte olarak) yer ayrılmıştır.

\section{KMO and Bartlett's Test}

Kaiser-Meyer-OlkinMeasure of SamplingAdequacy.

0,609

Bartlett's Test of Sphericity Approx. Chi-Square 18699,474

df 2278

Sig

0,000

KMO testi \%60,9 bulunmuştur. Bunun anlamı, istatistiksel tahmin ölçeği maddelerine faktör analizi yapılıp 
yapılamayacağını kontrol etmek amacıyla KMO değerinden ve Bartlett testinden yararlanılmıştır. 0,609>0,50 olduğundan veri grubumuzun faktör analizi için uygun olduğunu söyleyebiliriz. Yani gözlenen (gerçek) korelasyon katsayılarının büyüklüğü ile

kısmi korelasyon katsayılarının büyüklüğü karşılaştırılır. Bu testin değeri küçük çıkarsa, çift olarak değişkenler arasında ilişkinin diğer değişkenlerce açıklanmayacağını gösterir. $\mathrm{Bu}$ durumda da faktör analizine devam etmek doğru olmaz. Bizde KMO bir oran olup $\% 60$ in üstünde olması arzulanır. Bizde $\% 60,9$ \%61 dir. KMO aşağıdaki formülle hesaplanmaktadır.

$$
K M O=\frac{\sum_{i \neq j} \sum r_{i j}^{2}}{\sum_{i \neq j} \sum r_{i j}^{2}+\sum_{i \neq j} \sum a_{i j}^{2}}
$$

Burada $\mathrm{r}_{\mathrm{j}} \mathrm{j}$, i ile j no'lu değişkenler arasındaki basit korelasyon katsayısını gösterir. Paydadaki aij ise yine i ve j no'lu değişkenler arasındaki kısmi korelasyon katsayısını göstermektedir. Değişkenler arasında istenen derecede korelasyon vardır. Bartlett testi anlamlıdır. H0 reddedilir, örnek büyüklüğünün yeterli olduğu kabul edilmiştir. Ayrıca burada Faktör sayısının belirlenmesi, faktör modelinin kurulması ve asal bileşenler teorisine fazla değinmeyeceğiz. Ciddi ve derinlikli bir veri için hemen sonuçlara başvurup yorumları sizinle paylaşmak istiyoruz. Aşağıdaki Tablo 1'e bir göz atalım.

Tablo 1. Özdeğer (Eigenvalues) istatistiğine bă̆lı faktör sayısı ve varyans yüzdeleri

\begin{tabular}{|c|c|c|c|c|c|c|c|c|c|}
\hline \multirow[t]{2}{*}{ Component } & \multicolumn{3}{|c|}{ Initial Eigenvalues } & \multicolumn{3}{|c|}{$\begin{array}{c}\text { Extraction Sums of Squared } \\
\text { Loadings }\end{array}$} & \multicolumn{3}{|c|}{$\begin{array}{l}\text { RotationSums of } \\
\text { SquaredLoadings }\end{array}$} \\
\hline & Total & $\begin{array}{c}\% \text { of } \\
\text { Variance }\end{array}$ & $\begin{array}{c}\text { Cumulative } \\
\%\end{array}$ & Total & $\begin{array}{c}\% \text { of } \\
\text { Variance }\end{array}$ & $\begin{array}{c}\text { Cumulative } \\
\%\end{array}$ & Total & $\begin{array}{c}\% \text { of } \\
\text { Variance }\end{array}$ & $\begin{array}{c}\text { Cumulative } \\
\%\end{array}$ \\
\hline 1 & 4,957 & 7,290 & 7,290 & 4,957 & 7,290 & 7,290 & 3,415 & 5,023 & 5,023 \\
\hline 2 & 3,778 & 5,556 & 12,846 & 3,778 & 5,556 & 12,846 & 3,049 & 4,485 & 9,507 \\
\hline 3 & 3,265 & 4,801 & 17,647 & 3,265 & 4,801 & 17,647 & 2,985 & 4,390 & 13,898 \\
\hline 4 & 2,934 & 4,315 & 21,962 & 2,934 & 4,315 & 21,962 & 2,709 & 3,984 & 17,881 \\
\hline 5 & 2,679 & 3,939 & 25,901 & 2,679 & 3,939 & 25,901 & 2,698 & 3,967 & 21,849 \\
\hline 6 & 2,249 & 3,307 & 29,208 & 2,249 & 3,307 & 29,208 & 2,372 & & \\
\hline 7 & 1,775 & 2,611 & 31,819 & 1,775 & 2,611 & 31,819 & 1,928 & & \\
\hline 8 & 1,690 & 2,486 & 34,304 & 1,690 & 2,486 & 34,304 & 1,917 & & \\
\hline 9 & 1,599 & 2,352 & 36,656 & 1,599 & 2,352 & 36,656 & 1,871 & & \\
\hline 10 & 1,571 & 2,310 & 38,966 & 1,571 & 2,310 & 38,966 & 1,713 & & \\
\hline 11 & 1,464 & 2,153 & 41,118 & 1,464 & 2,153 & 41,118 & 1,672 & & \\
\hline 12 & 1,429 & 2,102 & 43,220 & 1,429 & 2,102 & 43,220 & 1,597 & & \\
\hline 13 & 1,309 & 1,925 & 45,145 & 1,309 & 1,925 & 45,145 & 1,426 & & \\
\hline 14 & 1,283 & 1,886 & 47,031 & 1,283 & 1,886 & 47,031 & 1,381 & & \\
\hline 15 & 1,225 & 1,802 & 48,833 & 1,225 & 1,802 & 48,833 & 1,360 & & \\
\hline 16 & 1,218 & 1,791 & 50,625 & 1,218 & 1,791 & 50,625 & 1,353 & & \\
\hline 17 & 1,177 & 1,731 & 52,356 & 1,177 & 1,731 & 52,356 & 1,314 & & \\
\hline 18 & 1,143 & 1,681 & 54,037 & 1,143 & 1,681 & 54,037 & 1,288 & & \\
\hline 19 & 1,104 & 1,624 & 55,661 & 1,104 & 1,624 & 55,661 & 1,283 & & \\
\hline 20 & 1,089 & 1,602 & 57,263 & 1,089 & 1,602 & 57,263 & 1,215 & & \\
\hline 21 & 1,057 & 1,555 & 58,818 & 1,057 & 1,555 & 58,818 & 1,181 & & \\
\hline 22 & 1,039 & 1,528 & 60,346 & 1,039 & 1,528 & 60,346 & 1,162 & & \\
\hline 23 & 1,006 & 1,479 & 61,825 & 1,006 & 1,479 & 61,825 & 1,150 & & \\
\hline 24 & ,973 & 1,431 & 63,256 & & & & & & \\
\hline 25 & ,963 & 1,417 & 64,673 & & & & & & \\
\hline 26 & ,956 & 1,406 & 66,078 & & & & & & \\
\hline 27 & ,908 & 1,335 & 67,414 & & & & & & \\
\hline 28 & ,897 & 1,319 & 68,733 & & & & & & \\
\hline 29 & ,892 & 1,311 & 70,044 & & & & & & \\
\hline$-I S S N: 2148$ & & & & & & & & 510 & \\
\hline
\end{tabular}




$\begin{array}{llll}\mathbf{3 0} & , 857 & 1,260 & 71,304 \\ \mathbf{3 1} & , 835 & 1,228 & 72,532 \\ \mathbf{3 2} & , 808 & 1,189 & 73,720\end{array}$

Hesaplanan communality (yükleyici) faktörlerin 1 olması beklenir. Bu değer yukarıda ifade ettiğimiz gibi aynı zamanda çıtı tablosunda ortak faktör (common factor) tarafindan açıklanan varyansın oransal payını (katkısını) gösterir. Ancak 1'den büyük özdeğeri olanlarla ilgilenmek gerekir. Toplam varyans 68 faktörle açıklanır ki değişken sayısı da zaten 68 dir. Birinci faktör toplam varyans1 4,957 özdeğeri ile açıklar. Yani toplam varyansın \%7,290'ünü oluşturur. İkinci faktör ise toplam varyansın 3,778 özdeğeri ile açıklar. 23. faktör 1,006 özdeğeri ile açılar. Ve 23. faktör birikimli olarak toplam varyansın $\% 61,825$ ini oluşturur. $\mathrm{Bu}$ değişkenler 23 faktör altında asal bileşenler tarafından istatistik açısından yeterli bulunmuştur. Çünkü 23 ten sonraki değişkenlerin açıklanan gücü ancak \%38,175 bulunmuştur.

İkinci elde ettiğimiz 2. tabloda 1. Faktör sütunu içinde en yüksek korelasyonlu değişkenler kümesi toplanmış olduğunu gördük. Şimdi bunlardan söz edelim

Rotated Component Matrix'e bakıldığında rotasyon işlemi sonucunda faktör 1 altında toplanan değişkenlerin daha yüksek korelasyonlarla toplandığını görmek açısından ilginç bir sonuç varsayılır. Şöyle ki VAR58 (yaşanabilecek ekonomik sıkıntılar) yine 1. sirada fakat Component Matrix'indeki korelasyon değeri 0,624 iken burada 0,806 $(\% 80,6)$ dir. Bu kez VAR 55 (salgin süreci sonunda iş bulmada karşılaşılacak zorluklar) ikinci sırayı yine 0,801 gibi yüksek bir korelasyonla yerini almıştır. Component Matrix'te bu oran 0,603 idi ve üçüncü sıradaydı. Component Matrix'te beşinci sıradaki VAR56 (salgın sürecinde işini kaybetme kaygısı) değişkeni RotatedMatrix'te üçüncü sırada bulunuyor ve 0,776 gibi oldukça yüksek bir korelasyonla. Daha önceki Component Matrix'te ikinci sirada olan VAR57 burada dördüncü sıraya öteleniyor. Yoruma gelince "Salgin süreci sonunda iş bulmada karşılaşıllacak zorluklar" öne geçiyor [VAR 55], COVID-19'a yakalanma korkusu daha alt siraya inmektedir. Psikolojik olarak irdeleme yapıldığında verimizin gerçeklere $1 s ̧ ı k$ tutması açısından çok önemli tahminlere ulaştı̆̆ görülmektedir. Component Matrix'te dördüncü sıradaki VAR59 (Aile Bireylerine COVID-19 bulaştırma kaygısı) korelasyonu 0,529 iken Rotated Component Matrix'te beşinci sıraya öteleniyor fakat korelasyon 0,667 dir. Aile bireylerine COVID-19'u bulaştırma kaygısının ötelenmesi tedavilerin başarılı geçtiği vaka sayılarının pandemi başlangıcında az olmasına bağlanabilir. Sonuçlara bakıldığında her iki faktör analizi yöntemiyle değişken grubu değişmemektedir. Fakat sırada korelasyonla ilişkili sıralama değişmektedir. $\mathrm{Bu}$ da verileri temsil eden değişkenlerle arasında güçlü bir bağlantı olduğunu göstermektedir.

Şimdi bu yöntemlerde 2. etkili faktör (Faktör 2) içindeki ilişkisi yüksek değişkenleri yorumlamaya çalışalım. Component Matrix'te (yükleyici faktör matrisinde) en yüksek korelasyon VAR6 (Sistemik bir hastalık var mı? sorusuna verilen yanıt 0,872$)$ değişkenine verilen yanıtlar ise VAR8 $(0,611) \mathrm{e}$ oranla (korelasyonla) "yüksek tansiyon" olarak yantlanmıştır. Çok önemli bir sonuç daha önce 65 yaş üstü için varılan kısıtları destekler nitelikte bir önemli etken olarak karşımıza çıkmaktadır. Aynı analiz Rotated Component Matrix ile yapıldığında Faktör 2 de toplanan değişkenler çok önemli bir grup oluşturmuşlardır. Salgın sürecinde COVID-19 etkeni ile enfekte olma riski derecelerini değerlendiriniz başlığı altında verilen yanıtları içeren değiş̧kenler içinde en yüksek korelasyonlu değiş̧kenler VAR23 (şoför) $(0,785)$, VAR22 (kurye) $(0,763)$, VAR21 (kasiyer) $(0,717)$, VAR24 (apartman görevlisi) $(0,70)$ ve VAR20 (polis memuru) $(0,655)$ dir. Mükemmele yakın, istatistiksel yönden güçlü ve anlam olarak pandemiyi en iyi niteleyen grup olarak karşımıza çıkmaktadır.

NEW FILE.
DATASET NAME DataSet2 WINDOW=FRONT.
DATASET ACTIVATE DataSet1.
NEW FILE.
DATASET NAME DataSet3 WINDOW=FRONT.
DATASET ACTIVATE DataSet1.
NEW FILE.


Tablo 2. Faktör Grupları

\begin{tabular}{|c|c|c|c|c|c|c|c|c|c|c|c|c|c|c|}
\hline & \multicolumn{14}{|c|}{ Rotated Component Matrix ${ }^{3}$} \\
\hline & \multicolumn{14}{|c|}{ Component } \\
\hline & 1 & 2 & 3 & 4 & 5 & 6 & 7 & 8 & 9 & 10 & 11 & 12 & 13 & 14 \\
\hline VAR58 & 808 & -123 & .032 &, 063 & .014 &,- 005 & $-0,046$ & -.027 & .068 & 084 & .022 & 038 & 085 & .002 \\
\hline VAR55 &, 801 &., 059 &, 043 &, 036 & .008 &, 042 & .031 & .038 & .035 &, 185 & .001 & -133 & .065 & .062 \\
\hline VAR56 &, 776 & .000 & .050 &., 010 & .054 & .020 & .041 & .072 &,- 128 &, 042 &, 008 & -177 & 028 & .017 \\
\hline VAR57 &, 671 &,- 069 &, 055 &, 078 & 029 &,- 015 &, 049 &,- 069 & 010 &, 102 &, 042 &, 121 &, 040 &, 024 \\
\hline VAR59 & 667 & -048 &,- 009 & .010 & 038 & -.053 & .032 & -101 & .076 & ,151 & 073 & 285 & 032 & -.053 \\
\hline VAR23 & $; 014$ & .785 & 0,031 & .004 & 003 & .033 & .009 & .014 & .028 & ,002 & .027 & .014 & .043 & .021 \\
\hline VAR22 &,- 031 & .763 &,- 007 &., 052 & 001 & .020 &, 055 & -.009 & .063 &, 059 & .010 &., 060 &., 049 & -011 \\
\hline VAR21 &., 075 & .720 &., 059 &, 040 & .056 &,- 024 &, 023 & .040 & .012 & .053 & .003 & 045 & .074 & .010 \\
\hline VAR24 & .043 & .700 & .052 &, 014 & , 105 &, 005 & .006 & -.075 & -.028 & .014 & .002 &, 175 & 103 & 033 \\
\hline VAR20 & $=077$ & 654 & :099 & $: 004$ & 038 & $=001$ & $=024$ & .148 & .045 & 055 & .058 & $=021$ & 0,041 & 018 \\
\hline VAR27 &, 035 &,- 092 & .805 &, 010 & .028 &,- 034 &, 027 & -.088 & .074 &, 050 & -033 &, 031 &, 025 & -141 \\
\hline VAR28 & .016 & .030 & 760 & .027 & .031 & .064 & .028 & .016 &., 006 & 030 & .037 & 010 & .040 & .116 \\
\hline VAR26 &, 012 & 011 &, 749 &, 042 & .059 &., 044 &, 016 & .033 & 109 &, 061 & .059 & .069 &, 020 & -026 \\
\hline VAR 30 &, 098 & $=056$ &, 602 &, 013 & .089 &., 081 &, 075 & .070 & $=, 030$ &, 059 &, 083 & -311 &, 037 &, 123 \\
\hline VAR29 & 024 & .093 & .550 & 000 & .033 & .024 & .011 & .057 & .017 & 147 & .260 &, 110 & 063 & -119 \\
\hline VAR60 & $=030$ & -039 &, 026 &, 771 & .020 &, 113 & $;, 065$ &, 009 &, 047 &, 064 &, 004 &, 073 & ,161 & .042 \\
\hline VAR61 &, 005 & -.033 &, 029 &, 756 &, 102 &, 046 & ;119 & .030 &, 042 &, 071 &, 085 &, 069 &, 039 & 061 \\
\hline VAR67 & -108 & .043 &,- 021 & -600 & .027 &,- 013 & , 106 & .095 & .075 &, 023 & .094 & -097 & -104 & -048 \\
\hline VAR6A & 132 & .026 &, 002 & 585 & .065 & .069 & .084 & .035 & -132 & 040 & 016 & .045 &, 120 & .028 \\
\hline VAR66 &, 048 & .019 &,- 001 &, 513 & -053 &, 004 & $;, 078$ &, 031 &,- 179 &, 006 &, 012 & -251 &,- 048 &,- 175 \\
\hline VAR63 & .030 &, 003 & .009 &, 385 & .061 & .078 &, 015 & .087 &,- 152 &, 002 & .022 & -243 & , 131 & -236 \\
\hline VART &, 038 & .008 & $=023$ &, 015 & .700 & .014 &, 002 & .021 & $-0,047$ &, 032 & .050 & -013 &, 030 & .058 \\
\hline VAR9 & 097 & 001 &, 026 &, 087 & 681 & 046 &, 002 & .054 &, 008 & 133 & .018 & 022 & .029 & .016 \\
\hline VAR\& & DBQ & $-0 \cap B$ & -004 & -004 & 594 & 286 & 0.59 & $-0>9$ & -059 & -088 & -069 & -106 & 087 & -067 \\
\hline
\end{tabular}

Verilerin uyumluluğunu gösteren KMO and Bartlett's Testi ise en az \%50 olması gerekirken çok daha yüksek \%81,2 olarak sonuçlanmıştır.

Component

\begin{tabular}{|c|c|c} 
& 1 & 2 \\
\hline VAR58 & $\mathbf{8 2 4}$ &,- 118 \\
\hline VAR55 & $\mathbf{, 8 0 6}$ &,- 055 \\
\hline VAR56 & $\mathbf{, 7 6 3}$ &, 001 \\
\hline VAR57 & $\mathbf{, 7 5 1}$ &,- 067 \\
\hline VAR59 & $\mathbf{, 7 2 7}$ &,- 035 \\
\hline VAR23 &,- 019 & $\mathbf{, 7 8 9}$ \\
\hline VAR22 &,- 062 & $\mathbf{, 7 7 5}$ \\
\hline VAR21 &,- 050 & $\mathbf{, 7 3 3}$ \\
\hline VAR24 &,- 043 & $\mathbf{, 7 0 6}$ \\
\hline VAR20 &,- 075 & $\mathbf{, 6 7 3}$ \\
\hline
\end{tabular}

Tablo 3. Döndürülmüsş Komponent Matrisi

\begin{tabular}{|c|c|c|c|c|c|c|c|c|c|c|c|}
\hline & 1 & 2 & 3 & 4 & 5 & 6 & 7 & 8 & 9 & 10 & 11 \\
\hline VAR58 &, 806 &,- 125 &, 030 &, 065 &,- 015 &,- 006 &,- 042 &,- 027 &, 067 & ,085 &,- 022 \\
\hline VAR55 &, 801 &,- 059 & ,043 & ,036 &,- 008 &, 042 &, 030 & ,038 &,- 035 & , 185 & ,000 \\
\hline VAR56 &, 776 & ,000 & ,050 &,- 012 &,- 054 & ,021 & ,039 & ,072 &,- 127 & ,042 & ,008 \\
\hline VAR57 &, 672 &,- 067 & ,057 & ,079 &, 030 &,- 015 &,- 053 &,- 088 &, 012 & ,101 & 042 \\
\hline VAR22 &,- 031 & ,763 &,- 006 &,- 053 & ,001 &, 020 &, 055 &,- 009 & ,064 &,- 059 &,- 010 \\
\hline VAR21 &,- 075 &, 717 &,- 062 &,- 035 &, 055 &,- 027 &, 028 & ,039 & ,010 &, 051 &,- 001 \\
\hline VAR24 &,- 043 & ,700 &, 054 &, 012 &,- 105 & ,007 &,- 007 &,- 075 &,- 028 &,- 013 &,- 004 \\
\hline
\end{tabular}


European Journal of Science and Technology

\begin{tabular}{|c|c|c|c|c|c|c|c|c|c|c|c|}
\hline VAR20 &,- 076 & ,655 &,- 098 &,- 004 &, 038 &,- 001 &,- 027 & ,148 & ,046 &, 055 &,- 059 \\
\hline VAR27 & ,036 &,- 091 &, 805 &, 010 &, 029 &,- 034 &, 025 &,- 088 &, 075 &, 049 &,- 033 \\
\hline VAR28 & ,016 &, 028 & ,759 &,- 024 &,- 031 &, 063 & ,032 &,- 016 &,- 007 &, 029 &,- 036 \\
\hline VAR26 & ,013 & ,012 & ,749 &, 041 &, 060 &,- 043 & ,014 &,- 033 &, 110 &,- 060 &, 059 \\
\hline VAR30 & ,098 &,- 056 & ,603 & ,011 &,- 089 &,- 080 &,- 077 &, 070 &,- 029 &,- 059 &, 084 \\
\hline VAR25 & ,036 &,- 056 & ,602 &, 023 &,- 037 & ,004 &,- 105 &, 025 &,- 071 &,- 020 &, 105 \\
\hline VAR29 &,- 023 &,- 094 &, 551 & ,002 &,- 032 &,- 024 &,- 009 &,- 057 &,- 015 &, 145 &,- 258 \\
\hline VAR60 &,- 030 &,- 039 &, 024 &, 774 &,- 021 & ,112 &,- 062 &, 010 &, 044 &, 062 &, 006 \\
\hline VAR61 & ,006 &,- 033 &, 027 & ,759 & ,101 &, 044 &,- 116 &,- 030 & ,038 &, 070 & ,086 \\
\hline
\end{tabular}

\section{KMO and Bartlett's Test}

Kaiser-Meyer-Olkin Measure of Sampling Adequacy. , 790

Bartlett's Test of Sphericity Approx. Chi-Square

\begin{tabular}{lr}
\hline df & 4545,638 \\
\hline Sig. & 105 \\
\hline
\end{tabular}

\section{Communalities}

\begin{tabular}{lr|r} 
& Initial & Extraction \\
\hline VAR23 & 1,000 &, 623 \\
\hline VAR22 & 1,000 &, 607 \\
\hline VAR21 & 1,000 &, 540 \\
\hline VAR24 & 1,000 &, 509 \\
\hline VAR20 & 1,000 &, 460 \\
\hline VAR27 & 1,000 &, 666 \\
\hline VAR28 & 1,000 &, 579 \\
\hline VAR26 & 1,000 &, 608 \\
\hline VAR30 & 1,000 &, 410 \\
\hline VAR25 & 1,000 &, 384 \\
\hline VAR58 & 1,000 &, 693 \\
\hline VAR55 & 1,000 &, 653 \\
\hline VAR56 & 1,000 &, 582 \\
\hline VAR57 & 1,000 &, 569 \\
\hline VAR59 & 1,000 &, 532 \\
\hline
\end{tabular}

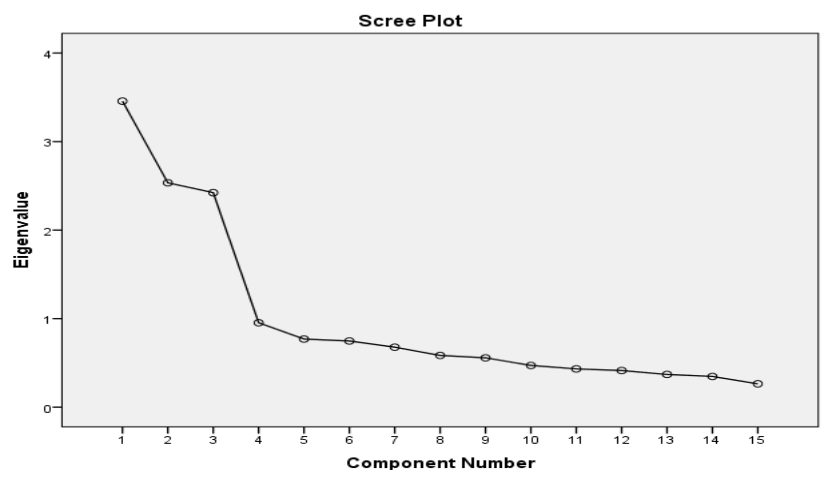

Component

\begin{tabular}{lr|r|r} 
& 1 & \multicolumn{1}{c}{2} & \multicolumn{1}{c}{3} \\
\hline VAR58 &, $\mathbf{8 2 3}$ &,- 117 &, 032 \\
\hline VAR55 &, 805 &,- 052 &, 044 \\
\hline VAR56 &, 762 &, 004 &, 043 \\
\hline VAR57 &, 749 &,- 066 &, 058 \\
\hline VAR59 &, 728 &,- 037 &,- 009 \\
\hline VAR23 &,- 019 &, $\mathbf{7 8 8}$ &,- 035 \\
\hline VAR22 &,- 064 &, 776 &,- 009 \\
\hline VAR21 &,- 046 &, 729 &,- 080 \\
\hline VAR24 &,- 048 &, 710 &, 048 \\
\hline VAR20 &,- 070 &, 666 &,- 104 \\
\hline VAR27 &, 064 &,- 086 &, $\mathbf{8 0 9}$ \\
\hline VAR26 &,- 003 &, 008 &, 779 \\
\hline VAR28 &, 021 &, 045 &, 759 \\
\hline VAR30 &, 055 &,- 070 &, 634 \\
\hline VAR25 &, 016 &,- 063 &, 616 \\
\hline & & & \\
\hline
\end{tabular}

Extraction Method: Principal

Component Analysis.

Rotation Method: Varimax with

Kaiser Normalization.

a. Rotation converged in 4 iterations. 
Tablo 4. Anket Sorularının Açıklaması

\begin{tabular}{|c|c|c|}
\hline & Initial & Extraction \\
\hline sira no & 1,000 & ,577 \\
\hline yaş & 1,000 & ,563 \\
\hline cinsiyetiniz & 1,000 & ,700 \\
\hline eğitim durumunuz & 1,000 & ,591 \\
\hline mesleğiniz & 1,000 & ,486 \\
\hline sistemik bir hastalığınız var mı? & 1,000 & 690 \\
\hline diyabet & 1,000 & ,577 \\
\hline yüksek tansiyon & 1,000 & ,588 \\
\hline kolesterol & 1,000 &, 595 \\
\hline solunum yetersizliği & 1,000 & 697 \\
\hline kalp & 1,000 & ,661 \\
\hline tiroid & 1,000 & ,688 \\
\hline diğer & 1,000 & ,682 \\
\hline bir hastalık yok & 1,000 &, 801 \\
\hline yaşadığınız il & 1,000 &, 598 \\
\hline $\begin{array}{l}\text { KOVID-19 salgının ne kadar tehlikeli olduğunu } \\
\text { düşünüyorsunuz? }\end{array}$ & 1,000 & ,431 \\
\hline enfekte olma risk & 1,000 & ,544 \\
\hline Öğretmen & 1,000 & ,566 \\
\hline Sağlık çalışanı & 1,000 & ,568 \\
\hline Polis & 1,000 &, 518 \\
\hline Kasiyer (alışveriş merkezi, market çalışanı vb.) & 1,000 & ,618 \\
\hline Kurye (yiyecek, kiyafet vb. taşıyan) & 1,000 & ,623 \\
\hline Şoför & 1,000 & ,642 \\
\hline Apartman görevlisi & 1,000 & ,626 \\
\hline Alışveriş merkezinde bulunmak & 1,000 & ,445 \\
\hline Toplu taşıma aracına binmek & 1,000 & 617 \\
\hline $\begin{array}{l}\text { Sosyal etkinliklere katılmak (arkadaşla } \\
\text { buluşma,sinema vb.) }\end{array}$ & 1,000 & ,731 \\
\hline Şehirlerarası seyahat etmek & 1,000 & 680 \\
\hline Açık havada spor yapmak & 1,000 & ,576 \\
\hline Sağlık kuruluşuna gitmek & 1,000 &, 545 \\
\hline Evinizde 60 yaş üstü yakınınız var mı? & 1,000 & ,571 \\
\hline Eldiven takmak & 1,000 & ,514 \\
\hline Yüze maske takmak & 1,000 &, 584 \\
\hline El hijyenine dikkat etmek & 1,000 & ,577 \\
\hline Selamlaşma esnasında temastan kaçmak & 1,000 & ,495 \\
\hline Toplu kullanıma açık tuvaletleri kullanmak & 1,000 &, 529 \\
\hline S1v1 veya katı el sabunu & 1,000 & ,597 \\
\hline Kolonya & 1,000 & ,608 \\
\hline İlaç ihtiva eden sıvı el dezenfektanı & 1,000 & ,574 \\
\hline
\end{tabular}


European Journal of Science and Technology

\begin{tabular}{|c|c|c|}
\hline Islak mendil & 1,000 &, 541 \\
\hline Aş1 & 1,000 & ,608 \\
\hline kilo değişiminiz & 1,000 &, 562 \\
\hline karbonhidrat-1 & 1,000 &, 840 \\
\hline vitamin-2 & 1,000 & ,843 \\
\hline hazır gida-3 & 1,000 & ,611 \\
\hline daha sağlıklı beslen-4 & 1,000 &, 823 \\
\hline beslenme alışkanlığım değişmedi & 1,000 & ,888 \\
\hline Televizyon seyretme & 1,000 & ,664 \\
\hline Kitap okuma & 1,000 &, 581 \\
\hline (Facebook, WhatsApp vb.) & 1,000 &, 539 \\
\hline Hobi faliyetleri (ahşap boyama, yapboz vb.) & 1,000 &, 536 \\
\hline Spor aktiviteleri & 1,000 & ,640 \\
\hline $\begin{array}{l}\text { Eğitim sisteminde uygulanan interaktif eğitime uyum } \\
\text { kaygısı }\end{array}$ & 1,000 & ,786 \\
\hline $\begin{array}{l}\text { Sinav tekniklerinde yaşanabilecek değişimlere } \\
\text { duyulan kayg1 }\end{array}$ & 1,000 & ,834 \\
\hline Salgın süreci sonunda iş bulmada karşılacak zorluklar & 1,000 & ,749 \\
\hline Salgın sürecinde işini kaybetme kaygısı & 1,000 & ,709 \\
\hline KOVID-19'a yakalanma korkusu & 1,000 & ,704 \\
\hline Yaşanabilecek ekonomik sıkıntılar & 1,000 & ,706 \\
\hline Aile bireylerine KOVID-19 bulaştırma kaygısı & 1,000 & ,655 \\
\hline uyku bozukluğu & 1,000 & ,686 \\
\hline Rüya içeriklerinin bozulması & 1,000 & ,654 \\
\hline yalnız kalma korkusu & 1,000 & ,357 \\
\hline kabızlık & 1,000 & ,490 \\
\hline öfke & 1,000 & ,489 \\
\hline sürekli el y1kama & 1,000 & ,728 \\
\hline midede hazımsızlık & 1,000 &, 505 \\
\hline Bir etkisi yok & 1,000 & ,708 \\
\hline $\begin{array}{l}\text { KOVID-19 salgını gelecek için yaptığınız } \\
\text { planlarınızın gerçekleşmesini }\end{array}$ & 1,000 & ,476 \\
\hline
\end{tabular}




\section{Sonuç}

Bu değişken grupları FAKTÖR 1 olarak VAR58, 55, 56, 57, 59 değişkenlerinin sırasıyla "ekonomik sıkıntı", "covid-19'a yakalanma korkusu", "iș bulmadaki zorluklar", "aile bireylerine covid-19 bulaştırma kaygısı" ve "işini kaybetme" olarak 68 değisskenden yüksek bir korelasyonla ortaya çıkartılması, ikinci önemli faktör olarak da FAKTÖR 2 (VAR23, 22, 21, 24, 20)olarak "en yüksek bulaş riski taşıyan meslek gruplarını" işaret etmesi ihmal edilmeyecek ve üzerinde dikkatle durulması gereken değişsen gruplarını ortaya çıkarmıştır. Hatta Faktör 3 ile de mükemmele yakın bir kümeleme ile ortaya çıkan (VAR27, 28, 26, $30,25)$ değişkenlerdeki ortak temanın "aktivite sıkıntısı çekme, aktivite yaşama zorluğunun" olması çalışmamızın çok boyutlu bir istatistiksel çalışma hedefini yakaladığını göstermektedir. Böylece 68 bağımsız değişken içinden 15 değişken yani \%25'lik değişken grubunun salgında etkin rol aldığı gösterilmiş oldu.

Önemli bir çalışma daha yaptık. Üç faktör altındaki bu değisskenleri kendi aralarında bir rotasyona tabi, her faktörün kendi içinde en yüksek korelasyona sahip üç değişkeni ölçmek

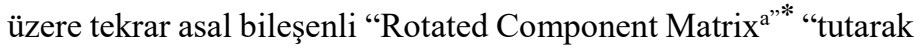
etkinliklerin derecelerini ölçtük. Uygunluk testi KMO'nun \%79 gibi çok yüksek olması yanında bilhassa grafikte de görüldüğü gibi 5. değişken ile 15. değişken arasındaki dağılımın tek başına bir küme oluştuğunu rahatça gözlemleyebiliyoruz. Bu değişkenler "enfekte olma riski taşıyan meslekler" ve "aktivite sıkıntısı çekme, aktivite zorluğu" değişken toplulukları yalnız COVID-19 için değil de ileride hangi tür salgın olursa olsun dikkat edilmesi gereken en önemli faktörler olarak görülmektedir. . Yani yalnız covid-19 değil yaşamla beraber oluşacak her türlü bulaş için çalışma sonuçlarının mutlaka referans alınmasını umuyoruz. VAR58 ile "yaşanabilecek ekonomik sıkıntılar", "VAR23 ile "sürücüler şoförler" ve VAR27 ile de "aktivite sıkıntısı çekme" yaklaşı \% 80 doğrulukla 68 değişken içinden en etkin değişkenler olarak karşımıza bir kez daha çıkmışlardır.

\section{GET}

FILE $=$ 'C: $:$ Users $\mid$ user|Documents|Untitled1-ADNAN-

MÜZEYYEN.sav-VERILER.sav'.

DATASET NAME DataSet1 WINDOW=FRONT.

FACTOR

/VARIABLES VAR23 VAR22 VAR2 1 VAR24 VAR20 VAR27

VAR28 VAR26 VAR30 VAR25 VAR58 VAR55 VAR56 VAR57

VAR59

/MISSING MEANSUB

/ANALYSIS VAR23 VAR22 VAR21 VAR24 VAR20 VAR27

VAR28 VAR26 VAR30 VAR25 VAR58 VAR55 VAR56 VAR57

VAR59

/PRINT INITIAL KMO EXTRACTION ROTATION

/FORMAT SORT

/PLOT EIGEN

/CRITERIA MINEIGEN(1) ITERATE(25)

/EXTRACTION PC

/CRITERIA ITERATE(25)

/ROTATION VARIMAX

/SAVE REG(ALL)

$/$ METHOD=CORRELATION.

\section{Kaynakça}

[1]Birgül

Özgüvenç,

https://www.a3haber.com/2020/09/15/salgininpsikolojiketkileri-virüsleilgili-belirsizlik-depresyon-gibihastaliklara-zemin-hazirladi/. Erişim tarihi.10.08.2021

[2] Adnan Mazmanoğlu, Etkileşimsiz çapraz 2 faktörlü varyans analizi modellerinde matrislerle çözümleme, İstanbul University, Post graduate thesis, İstanbul, 1984.

[3] Johannes Petrus van de Geer, Introduction to Multivariate Analysis for the Social Sciences, W. H. Freeman, San. Francisco, 1971, pp. 85-92.

[4] Harold Hotelling, Analysis of a complex of statistical variables into principal components, Journal of Educational Psychology 24(6) (1933), 417-441.

[5] Adnan Mazmanoğlu, Faktör Analizi ve Bilgisayarlarda Modern F. A.'I Yöntemlerinin Kullanımı, Post Graduate Thesis, İstanbul University, 1979, p. 24-52.

[6] Centers for Disease Control and Prevention, Coronavirus Disease 2019 (COVID-19) How to Protect Yourself. (2020, 4 April)

https://www.cdc.gov/coronavirus/2019ncov/prepare/preventi on.htm.

[7] Centers for Disease Control and Prevention, Coronavirus Disease 2019 (COVID-19) How to Protect Yourself. (2020, 4 April) https://www.cdc.gov/coronavirus/2019-ncov/preventgetting-sick/prevention.html

[8] N. L. Zhong, W. Luo, H. M. Li, Q. Q. Zhang, X. G. Liu, W. T. $\mathrm{Li}$ and $\mathrm{Y}$. Li, Knowledge, attitudes, and practices towards COVID-19 among Chinese residents during therapidrise period of the COVID-19 outbreak: a quick online crosssectional survey, International Journal of Biological Sciences 16(10) (2020), 1745-1752. doi: 10.7150/ijbs.45221

[9] European Centre for Disease Prevention and Control, Rapid risk assessment: novel coronavirus disease 2019 (COVID-19) pandemic: increased transmission in the EU/EEA and the UK - sixth update, Access; 2020. https://www.ecdc.europa.eu/en/publications-data/rapidriskassessment-novelcoronavirusdisease-2019-covid-19pandemic increased.

[10] M. S. Wolf, M. Serper, L. Opsasnick, R. M. O'Conor, L. M. Curtis, J. Y. Benavente, G. Wismer, S. Batio, M. Eifler et al., Awareness, Attitudes, and Actions Related to COVID-19 Among Adults with Chronic Conditions at the Onset of the U.S. Outbreak 2020. Ann. Intern. Med. doi: 10.7326/M201239

[11] J. Brug, A. R. Aro, A. Oenema, O. Zwart, J. H. Richardus and G. D. Bishop, SARS Risk Perception, Knowledge, Precautions, and Information Sources, the Netherlands. Emerging Infectious Diseases 10(8) 2004. www.cdc.gov/eid

[12] G. Akdeniz, M. Kavakcı, M. Gözügök and A. Yalçınkaya, Survey of attitudes, anxiety status, and protective behaviors of the university students during the COVID-19 outbreak in Turkey, Frontiers Psychiatry 11 2020, Article 695. https://doi.org/10.3389/fpsyt.2020.00695

[13] European Centers for Disease Control and Prevention, Environmental Cleaning and Disinfection Recommendations, (2020, 5 April). Access address: https://www.cdc.gov/coronavirus/2019 ncov/community/organizations/ cleaningdisinfection.html 
[14] B.L. Zhong, W. Luo, H.M. Li, Q.Q. Zhang, X.G. Liu, W.T. $\mathrm{Li}$ and Y. Li. Knowledge, attitudes, and practices towards COVID-19 among Chinese residents during the rapid rise period of the COVID-19 outbreak: a quick online crossMultidimensional Modeling to Find the Most Efficient Factors ... 31 sectional survey, Int. J. Biol. Sci. 16(10) (2020), 1745-1752. doi:10.7150/ijbs.45221

[15] J. Yang, Y. Zheng, X. Gou, et al., Prevalence o comorbidities and its effects in patients infected with SARS-CoV-2: a systematic review and meta-analysis, Int. J. Infect Dis. (2020). doi: 10.1016/j.ijid.2020.03.017

[16] G. Singla, T. Singla and S. Singla, COVID-19 Pandemic - A literature review, International Journal of Research and Review 282(7) (2020), 282-293.

[17] S. S. Dickerson and M. E. Kemeny, Akut stres faktörleri ve kortizol yanıtları: Laboratuvar araştırmalarının teorik entegrasyonu ve sentezi, Explained in Psyschol. Boğa. 130 (2004), 355-391.

[18] Z. Karataş Sociallmpacts of COVID-19 Pandemic, Change and Empowerment, Türkiye Sosyal Hizmet Araştırmaları Dergisi 4(1) (2020), 3-17. https://dergipark.org.tr/tushad

[19] R. Aslan, Tarihten günümüze epidemiler, pandemiler ve covid-19, Göller Bölgesi Aylık Ekonomi ve Kültür Dergisi 8(85) (2020), 35-41.

[20] W. Li, Y. Yang, Z. H. Liu, Y. J. Zhao, O. Zhang, L. Zhang, T. Cheung and T. Y. Xiang, Progression of mental health services during the covid-19 outbreak in China, Int. J. Biol. Sci. 16(10) (2020), 1732-1738.

[21] Istituto Superiore di Sanità, Characteristics of SARS-CoV-2 patients dying in Italy, Report based on available data on April 29, 2020. 2020. https://www.epicentro.iss.it/en/coronavirus/bollettino/Report COVID-2019_29_april_2020.pdf (accessed May 5, 2020).

[22] Prevention and control of non-communicable diseases in the COVID-19 response. Published Online May 8, 2020. https://doi.org/10.1016/ S0140-6736(20)31067-9

[23] F. Amanat and F. Krammer, SARS-CoV-2 Vaccines: Status Report, Immunity 52(14) (2020), 583-589.

[24] J. J. V. Bavel, K. Baicker, R. Willer et al., Using social and behavioural science to support COVID-19 pandemic response, Nature Human Behaviour 4 (2020), 460- 471.

[25] S. J. Jung and J. Y. Jun, Mental Health and Psychological Intervention Amid COVID-19 Outbreak: Perspectives from South Korea, Yonsei Med. J. 61(4) (2020), 271-272. https://doi.org/10.3349/ymj.2020.61.4.271

[26] B. Pfefferbaum and C. North, Mental Health and the Covid19 Pandemic, TheNew England Journal of Medicine 383 (2020), 510-512. doi: 10.1056/NEJMp2008017

[27] Z. Şenol and F. Zeren, Coronavirus (COVID-19) and stock markets: the effects of the pandemic on the global economy, Eurasian Journal of Researches in Social and Economics (EJRSE), ASEAD CILT 7 SAYI 4 Yil 2020, S 1-16.

[28] Y. Zhang and Z. F. Ma, Impact of the COVID-19 pandemic on mental health and quality of life among local residents in Liaoning province, China: A crosssectional study, Int. J. Environ. Res. Public Health 17 (2020), 2381. doi:10.3390/ijerph17072381 www.mdpi.com/journal/ijerph.

[29] J. Guo, X. L. Feng and X. H. Wang, Coping with COVID19: Exposure to COVID-19 and negative impact on livelihood predict elevated mental health problems in Chinese adults, Int. J. Environ. Res. Public Health 17(11) (2020), 3857. Published online 2020 May 29. doi: 10.3390/ijerph17113857
[30] A. Wilder-Smith and D. O. Freedman, Isolation, quarantine, social distancing and community containment: pivotal role for old-style public health measures in the novel coronavirus (2019-nCoV) outbreak, J. Travel Med. 27 (2020), 1-4. doi: $10.1093 / \mathrm{jtm} / \mathrm{taaa} 020$. 